\title{
Mass Spectrometry Characterization of a Novel Insulin Mimetic Peptide s597
}

\author{
Natalia Mesonzhnik ${ }^{1}$, Grigory Krotov ${ }^{2}$ and Svetlana Appolonova ${ }^{1^{*}}$ \\ ${ }^{1}$ Institute of Pharmacy and Translational Medicine, Sechenov First Moscow State Medical University, 119991 Moscow, Russia \\ ${ }^{2}$ NRC Institute of Immunology FMBA of Russia, 115478, Moscow, Russia
}

"Corresponding author: Svetlana Appolonova, Institute of Pharmacy and Translational Medicine, Sechenov First Moscow State Medical University, Nakhimovsky Prospect 45, Moscow, Russian, Tel: +79032796571; E-mail: svetlana.appolonova@labworks.ru

Received date: March 29, 2017; Accepted date: May 22, 2017; Published date: June 07, 2017

Copyright: @ 2017 Mesonzhnik N, et al. This is an open-access article distributed under the terms of the Creative Commons Attribution License, which permits unrestricted use, distribution, and reproduction in any medium, provided the original author and source are credited.

\begin{abstract}
Novel peptide-based drugs have recently gained high popularity, especially among athletes seeking ways to enhance their performance. Although the World Anti-Doping Agency (WADA) has banned the use of any nonapproved for human therapeutic use pharmacological substances in Sports, a huge variety of such peptides with potential performance-enhancing properties are available for sale in the black market and in illegal online websites. The difficulty of determination of these molecules in biological fluids depending on their low concentrations and their similarity to endogenous compounds has boosted their use not only among athletes, but also in the amateurs' world.

The goal of this study was to perform the mass spectrometry characterization of a novel s597 peptide drug purchased via an online store. The study was carried out using nanoscale liquid chromatography/quadrupole Orbitrap mass spectrometry with accurate mass determination and sequence analysis for both intact drug and after its trypsinolysis. The purchased drug was found to be a peptide with 31 amino acid residues, intra-chain disulfide bond and modified by $\mathrm{C}$-terminal amide and $\mathrm{N}$-terminal acetyl groups. The proposed sequence was consistent with s597 peptide, designed to be used as insulin receptor ligand mimetic.
\end{abstract}

Keywords: s597; Peptide-based drugs; LC-HRMS/MS

\section{Introduction}

Selective insulin receptor modulators represent novel promising anti-diabetic drug candidates [1]. The concept to obtain low molecular weight target-specific ligands through combinatorial peptide strategies using phage display, followed by pharmaceutical optimization, has received much attention in drug discovery. Recently several groups of insulin mimetic peptides, generated from screening of phage display peptide libraries, were found to bind different sites on the Insulin Receptor (IR). Non-sequence homological peptides bound to two discrete «hotspots» on the defined sites of IR (site 1 and site 2), which correspond to contact sites involved in insulin binding according to a predicted «cross-linked» model $[2,3]$. It was shown that group of site 1 mimetic peptides were full agonist with low affinity or antagonists, whereas site 2 peptides were antagonist or inactive in terms of IR phosphorylation. Remarkably, the site 1 peptides also bound to the Insulin Growth Factor-1 Receptor (IGF-1R) in contrast to the site 2 peptides. Two different affinity-optimized site 1 (s371) and site 2 (s446) peptides were subsequently linked in all possible amino-tocarboxyl terminal orientation to make synthetic and recombinant homo- and heterodimers to create high specificity and potency IR modulator molecules. The heterodimers were designated as site 1 site 2 or site 2 site 1 , according to order of surrogate peptides and showed improved binding in comparison to monomers. In addition, their properties strongly depend on the orientation of docking peptide linkages: N-N or C-C (end-to-end orientation) peptides were weak or partial agonists (s454 and s456), C-N site 1site 2 peptide (s453) was an antagonist, and C-N site 2site 1 peptide (s455) was a full agonist. Since the optimal orientation for agonistic properties was site 2 site 1 , further optimization of the agonist sequence was performed by solid-phase synthesis. The changes made in the optimization process resulted in an improved peptide designated as s519, which is 36 a.a. single chain with one internal disulfide bridge. The peptide showed insulin-like activities in both in vivo and in vitro assays. Further investigation of s519 peptide revealed its most important features such as the increase of the receptor potency and affinity, including $\mathrm{N}$-terminal acetylation, and an amino acid replacing and deleting sequence GSLD at positions 21-24. The optimized peptide sequences, which show in vitro and in vivo activity comparable to insulin, were s597 and its des-acetyl variant s557 [4].

The important aspect of site2site1 dimers properties was relevant to the specificity of binding to the IR vs. closely related IGF-1R. IR and IGF-1R belong to the growth factor tyrosine-kinase receptor superfamily. Despite sequence similarities of about $41-84 \%$ (primary in alfa-subunit), IR and IGF-1 receptors determine different physiological functions. IR is associated with metabolic response while IGF-1R is involved in the process of cells growth and differentiation. IGF-1 and insulin competitively cross-react with IGF-1R and IR with weak binding affinity to each other's receptors. Thereby, each ligand can induce both types of effects [5]. Because of greater mitogenic activity associated with IGF-1R comparing to IR, the development of high specify molecular structures as insulin agonists is promising direction for antidiabetic drug discovery.

Recently, Jensen et al. found that a heterodimer (site 2-site 1 mimetic) s597 had a similar affinity as insulin to the IR [6]. It was reported that the insulin mimetic peptide activated the IR in a signaling manner significantly different from insulin. Activation of the 
IR by insulin led to receptor phosphorylation, internalization of the complex and phosphorylation of several intracellular protein substrates, including IRS1, IRS2 and Shc, followed by initiation of two major signaling cascades: the PI 3-kinase pathway (activation of glycogen synthesis) and MAPK (cell growth). In contrast, the peptide s597 was able to differentially activate the IR most likely due to the differences in receptor interaction, low phosphorylation of the IR and low degree of IR internalization. The mimetic peptide fully activated the PI 3-kinase pathway, but weakly activated (no phosphorylated ERK was detected) the Shc/ERK (MAPK) pathway resulting in full activation of glycogen synthesis, but less induction of gene expression and cell proliferation.

According to the present knowledge, MAPK signaling pathway plays important role in the induction of the mitogenic response. Therefore the difference in mitogenitic response between s597 and insulin results from different signaling more than from cross-talking with IGF-1R. This is supported by the finding that $s 597$ had no effect on phosphorylation of the IGF-1R, ERK1/2 or PKB in untransfected L6 cells [6] and by studies of the gene expression profile in IRtransfected L6 myoblasts after IR activation by insulin and s597, using microarray technology [7]. It was shown that almost half of the genes differentially regulated by $s 597$ and insulin were related with cell proliferation and growth. In short, many of the changes in selective gene expression or potency induced by insulin had a positive effect on cell growth and proliferation. In parallel, a half of the differentially regulated genes directly or indirectly interact with the genes involved with MAPK signaling pathway.

It is well known that the therapeutic use of insulin and its analogues is associated with typical problems of the biotechnological protein drugs such as high costs of production, mandatory administration via injection, instability in the bloodstream. An additional drawback of several insulin analogues is an enhanced mitogeniticity [8]. On these grounds, insulin mimetic peptides can be proposed as potential drug candidates in the treatment of diabetes due to their smaller size, simple structure and insulin-like activity with low mitogenic response, which are critical issues in the development of effective and safe pharmaceuticals.

Although the insulin mimetic compound s597 has so far not been approved for therapeutic use, it is easily accessible via Internet in the form of powder. In fact, this product is proposed by dealers to athletes in several web-based peptide selling sites and in Internet sport forums easily accessible in Russia as drugs to «improve performance via metabolic effect of insulin but without side mitogenic effect». However, the identification of 5597 peptide in online market products has not been reported to date. In the present paper, an LC-MS method for identification and structure characterization of this novel insulin mimetic peptide is reported, as well as its application on a product found in the Russian black market.

\section{Experimental}

\section{Chemical and reagents}

High purity water was prepared in-house using Milli-Q water purification system obtained from Merck KGaA (Darmstadt, Germany). HPLC grade methanol and acetonitrile were purchased from Thermo Fisher Scientific Inc. (Madrid, Spain) and Panreac (Barcelona, Spain) respectively. Extra pure acetic acid, formic acid and ammonium format were purchased from Fluka (Steinheim, Germany). DL-ditiothreitol ( $\geq 98 \%$, DTT) was purchased from Sigma-Aldrich (St Louis, MO, USA). Iodoacetamide ( $\geq 99 \%)$ was purchased from Sigma (St Louis, MO, USA).

\section{Solution preparation}

The content of a vial labelled as «s597» (allegedly, from «5 star peptides» manufacturer) was dissolved in water and $1 \mathrm{ml}$ of the stock solution at a nominal concentration of $0.5 \mathrm{mg} / \mathrm{ml}$ was extracted via SPE using Oasis HLB cartridges (Waters, Milford, MA, USA). After evaporation, the residue was reconstituted in $200 \mu \mathrm{L} 50 \% \mathrm{ACN}$ in $2 \%$ acetic acid aqueous solution. Prior to LC-MS analysis, the intact peptide solution was diluted 1:100 with the HPLC mobile phase. Alternatively, the peptide original solution was subjected to trypsin digestion at a 1:20 (w:w) enzyme:protein ratio (Promega, Madison, WI, USA) in $50 \mathrm{mM}$ ammonium bicarbonate (Merck, Germany). Hydrolysis was carried out overnight at $37^{\circ} \mathrm{C}$. Prior to digestion, cysteine residues were reduced with DTT followed by incubation with iodoacetamide in the dark.

\section{LC-HRMS/MS analysis}

The original sample and the tryptic digest were investigated by LCHRMS/MS using an Ultimate 3000 nano-LC system coupled with a hybrid quadrupole-Orbitrap "Q Exactive" (Thermo Scientific, Bremen, Germany). The LC trapping was performed on an Acclaim PepMap $100 \mathrm{~A}, \mathrm{C}-18(75 \mu \mathrm{m} \times 2 \mathrm{~cm}, 3 \mu \mathrm{m})$ column at $3 \mu \mathrm{l} / \mathrm{min}$ using an aqueous mobile phase containing $0.08 \%$ TFA, $2 \% \mathrm{ACN}$ and $0.1 \% \mathrm{FA}$. After $5 \mathrm{~min}$, the trap column was connected on-line with an analytical column Agilent Zorbax 300SB-C18 $(75 \mu \mathrm{m} \times 15 \mathrm{~cm}, 3.5 \mu \mathrm{m})$. The analytes were than eluted with a 40 min linear gradient (from B $5 \%$ to B 95\% ) at a flow rate of $0.350 \mu \mathrm{l} / \mathrm{min}$, where solvent A was HPLC grade water with $0.1 \% \mathrm{FA}$ and solvent $\mathrm{B}$ was $0.1 \% \mathrm{FA}$ in $\mathrm{ACN} / \mathrm{H}_{2} \mathrm{O}$ $(80 / 20 \mathrm{v} / \mathrm{v})$. One microliter of each sample was injected onto the LCMS system.

Mass spectrometry experiments were performed using a NanoFlex ion source (Thermo Scientific, Germany) equipped with a steel emitter, $2.1 \mathrm{kV}$ spray voltage, $275^{\circ} \mathrm{C}$ capillary temperature. S-lens level was set at 50. Q Exactive was operated in data-dependent acquisition mode. Full scan MS spectra were acquired with a resolution settings of 70000 (at $\mathrm{m} / \mathrm{z} 200$ ) or 35000 (at $\mathrm{m} / \mathrm{z}$ 200). For each full scan the three most intense ions were isolated and fragmented by $\mathrm{HCD}$, high resolution MS/MS spectra were acquired with a resolution settings of 35000 (at $\mathrm{m} / \mathrm{z} 200$ ) or 17500 (at $\mathrm{m} / \mathrm{z} 200$ ) (isolation width of $3 \mathrm{Da}$ ).

\section{Results and Discussion}

The product purchased from the Internet was analyzed using LCMS/MS in order to detect and identify the declared substance. The amino acid sequence of s597 reported by Jensen [1,2] consists of 31 amino acid residues in the following order: SLEEEWAQIECEVYGRGCPSESFYDWFERQL (one-letter code). In addition, the $\mathrm{C}$-terminal amide and the $\mathrm{N}$-acetyl modifications should be incorporated into the structure of the polypeptide molecule [1]. 


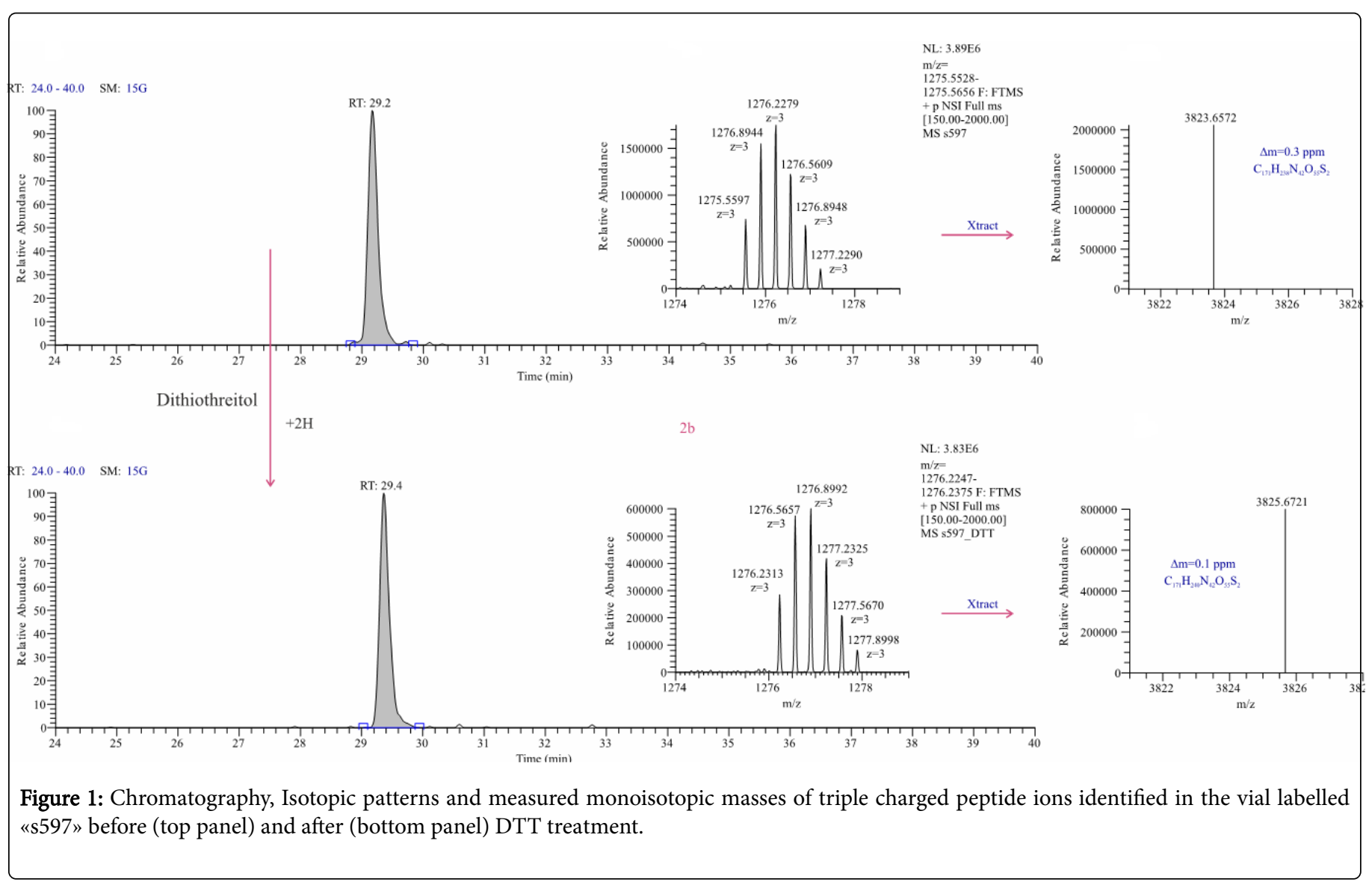

The charge state of the most abundant ion in the spectra of intact peptide found in the preparation labelled as «s597» was determined as $3+(\mathrm{m} / \mathrm{z} 1275.5597)$ and used for accurate molecular weight calculation and sequencing (Figures 1 and 2).

The experimentally measured monoisotopic mass value of the peptide was found to be approximately 2 Da lower than that of the theoretically calculated of the reported end-terminals modified peptide sequence, which contains two cysteine residues [1]. This mass difference was zeroed after the reducing treatment with DTT, thus suggesting that a disulphide bond was present in the s597 structure (Figure 1).

The product ion spectrum of the triple charge precursor ion contained the most abundant peaks associated with N-terminal fragments, relevant to the single charge $b$-ion and double charge $y$-ion series (Figure 2). A mass addition corresponding to an acetyl group was found on all observed b-ions, indicating the N-terminal localization of acetylation. The observed y-ions were consistent with a C-terminal leucine amidation.
The localization of an internal disulfide bond (C11-C18) was identified from the accurate mass differences between y1-y13 ions series (matched to the predicted mass) and y21-y30 (matched to the predicted mass minus two hydrogen atoms). Fragment ions within the disulfide bond region were either with very low intensity or not observed. After treatment with DTT the peak ions associated with the disulphide bond were shifted to higher mass, consistently with the addition of two hydrogen atoms during reduction.

To allow unambiguous structure assignment, tryptic digests of reduced and alkylated peptide were examined by LC-MS/MS. Two main tryptic fragments were observed in the LC-MS/MS data (Figure 3 ). The observed monoisotopic masses of peptides at $\mathrm{m} / \mathrm{z} 1020.4530$ $(2+)$ and $\mathrm{m} / \mathrm{z} 840.3434(2+)$ was consistent with those theoretically calculated for expected alkylated tryptic peptides of s597: Acetyl-S1R16 $\left(\mathrm{C}_{88} \mathrm{H}_{130} \mathrm{~N}_{22} \mathrm{O}_{32} \mathrm{~S}, 2038.8942 \mathrm{Da}, 0.3 \mathrm{ppm}\right)$ and G17-R29 $\left(\mathrm{C}_{76} \mathrm{H}_{98} \mathrm{~N}_{18} \mathrm{O}_{24} \mathrm{~S}, 1678.6722,0.06 \mathrm{ppm}\right)$. On the basis of the signature peptide identified at $2+$ charge states precursor ions the peptide was manually assigned. Mass accuracies of the peptides fragment ions were within $5 \mathrm{ppm}$ of the corresponding theoretical $\mathrm{m} / \mathrm{z}$ values. 


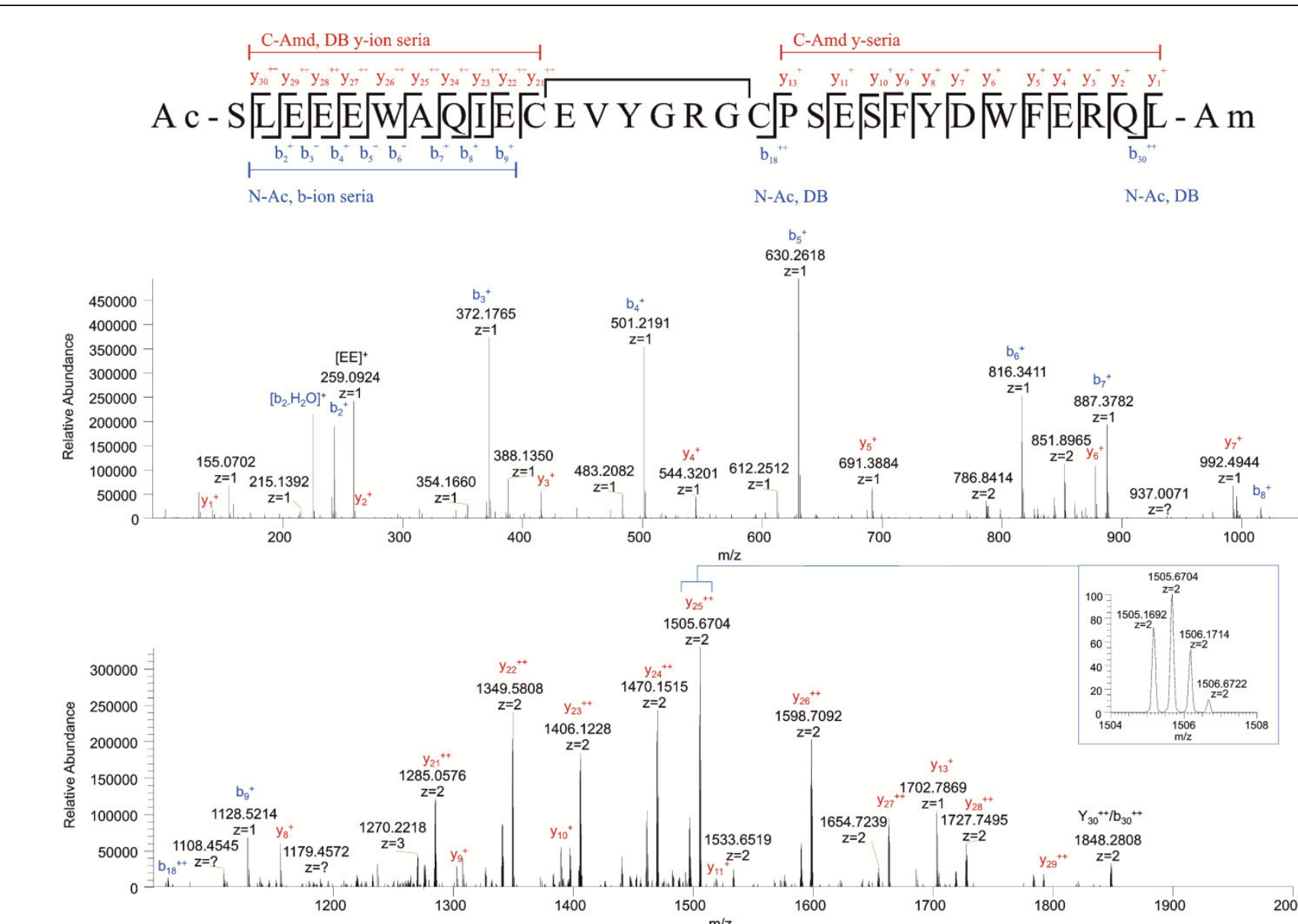

Figure 2: HCD spectra (NCE of 23) obtained from triply-charged precursor presented at split mass range of $100 \mathrm{~m} / \mathrm{z}-1050 \mathrm{~m} / \mathrm{z}$ (top panel) and $1050 \mathrm{~m} / \mathrm{z}-2000 \mathrm{~m} / \mathrm{z}$ (bottom panel) and fragments assignment of the intact peptide found in the preparation labelled as «s597». DB-disulfide bond, N-Ac-N-acetyl, C-Amd-C-amide. ${ }^{\star}$ Isobaric amino acids leucine and isoleucine designated according to the reported sequence [6].

The product ion spectra of carbamidomethylated peptide G17-R29 gave almost exclusively strong $y$-ion series at eleven of the twelve amide bond cleavages, while only one b-ion was observed, arising from cleavage of the $\mathrm{C} 2-\mathrm{P} 3$ bond (viz. b2-ion). Additional less informative fragment ions corresponding to neutral losses and internal fragments with $\mathrm{N}$-terminal proline residue were also observed in the spectrum. The derived amino acid sequence, GCPSESFYDWFER, was identical to the theoretical s597 tryptic peptide G17-R29.

Another s597 alkylated tryptic peptide, Ac-S1-R16, yielded complex fragmentation pattern consisting of dominated y-type ions intersecting with brief series of six b-ions. The sufficient sequence information allowed giving the amino acid sequence Ac-SLEEEWAQIECEVYGR that was consistent with what was theoretically expected. The location of $\mathrm{N}$-terminal serine acetylation in observed tryptic peptide was confirmed by characteristic mass shift for the b-ion species. It should be noted, that blocking the groups attached at the N- and C-ends of s597 peptide chain can improve the peptide stability; therefore these modifications might increase the biological activity of the insulin mimetic product. Incorporation of the N-terminal acetylation into the structure of s597 unacetylated variant (s557) led to an improvement of blood glucose lowering effect in rats [4]. Afterwards, a strategy for increasing the enzymatic stability of the IR-binding peptides (e.g. the s597 peptide) through enzymatic digestion analysis was devised. Using a series of relevant digestive enzymes the major cleavage sites of s597 were revealed by mass-spectrometry, and accordingly a novel optimized peptide, named s873, was created by introducing the unusual a.a. residues aminoisobutyric acid and diphenylalanine, and by replacing and adding several amino acids as well. IR affinity similar to insulin and greater stability as compared to s597 was experimentally found for the optimized peptide $s 873$ [9]. No other information about peptide 873 has been found in the available sources.

\section{Conclusion}

The studies of the of IR and IGF-1R activation mechanism which have been conducted in the recent years showed the critical points for the design of new insulin mimetic drugs. The group of the synthetic insulin mimetic peptides, including s597, shows a promising therapeutical potential both in vitro and in vivo studies. However, the lack of clinical trials remains question whether this peptide could have any clinical applications. Nerveless, s597 peptide is already on the market under the label "for research use only". 

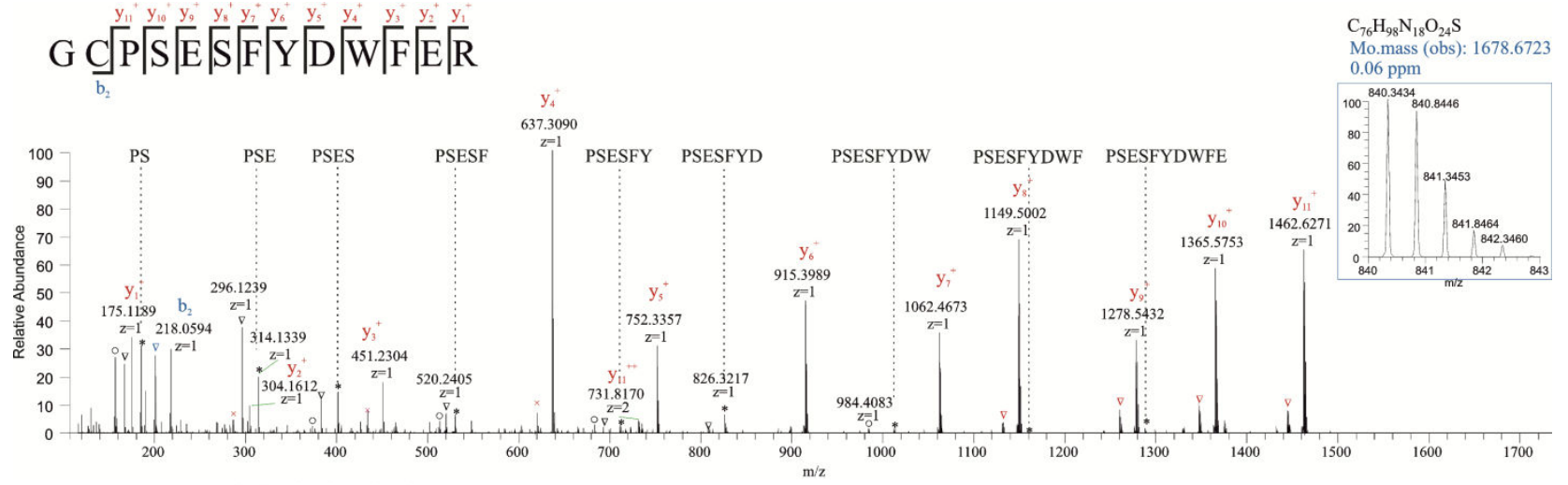

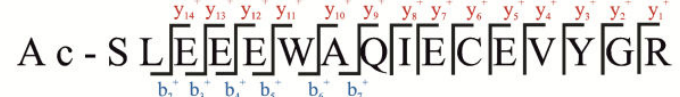

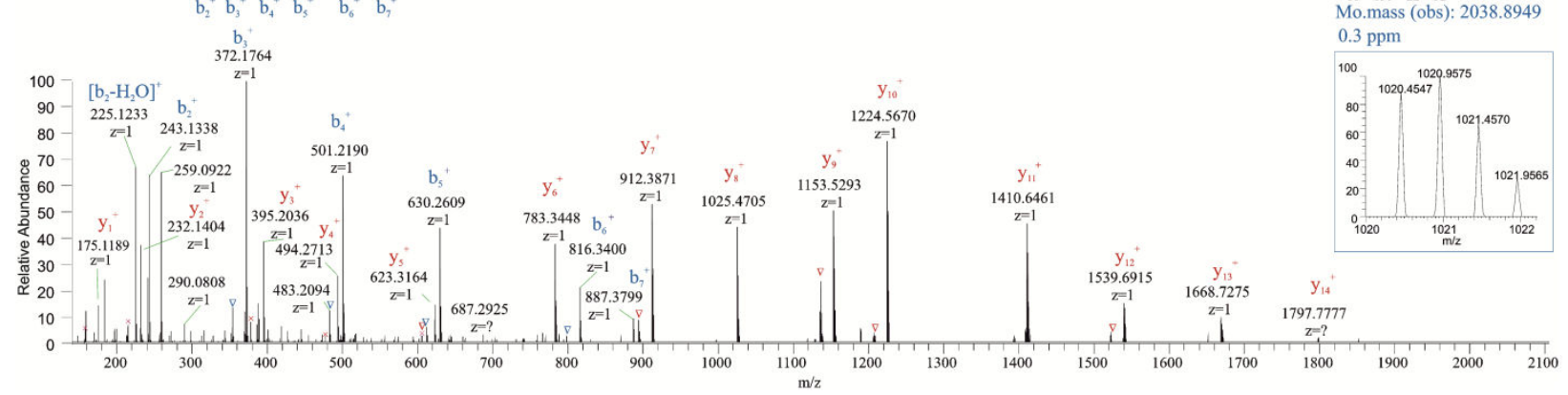

Figure 3: Isotopic patterns (inserts), observed monoisotopic masses and product ion spectra (NCE of 30) of doubly-protonated alkylated peptides Gly17-Arg29 (top panel) and Acetyl-Ser1-Arg16 (bottom panel) with spectrum-to-sequence assignment of tryptic peptides found in the preparation labelled as «s597» after enzymatic digestion. Additional observed ions arising through additional loss of $\mathrm{H}_{2} \mathrm{O} / \mathrm{NH}_{3}$ from the $\mathrm{b}$ and y ion species, and internal fragments are denoted as follow: inverted triangles-loss of $\mathrm{H}_{2} \mathrm{O}$; crosses-loss of $\mathrm{NH}_{3}$; circles-loss of CO; starshaped character-internal proline fragments.

The mass-spectrometric structure characterization of a sample of peptide 5597 (as declared) obtained from an "on line" distributor was performed on either the intact molecule or the fragments obtained after the trypsin digesting. The experimentally obtained LC- MS data and the observed fragmentation patterns from LC-MS/MS of the investigated substance were consistent with the authentic amino acid sequence of S597 insulin mimetic peptide consisting of 31 amino acid residues with disulfide bond, $\mathrm{C}$-terminal amide and $\mathrm{N}$-terminal acetyl modifications [6].

The reported data are particularly interesting also in the forensic environment because metabolic modulators including insulin are prohibited substances under Section S4 of the WADA Prohibited List [10], and insulin misuse by athletes have already been reported [11]. For this reason, novel insulin mimetic substances, as potential doping agents, should be monitored, even if, to date, only for preventive purposes. In fact, WADA has banned the use of any pharmacological non-approved substances under Section S0 of the WADA Prohibited List [10].

\section{References}

1. Blume AJ, Brandt J, Brissette R, Goldstein NI, Ostergaard S, et al. (2003) Insulin and igf-1 receptor agonists and antagonists. PCT Int Appl WO03070747.

2. Jensen M, Hansen B, De Meyts P, Schäffer L, Urso B (2007) Activation of the insulin receptor by insulin and a synthetic peptide leads to divergent metabolic and mitogenic signaling and responses. J Biol Chem 282: 35179-35186.

3. Jensen M, Palsgaard J, Borup R, De Meyts P, Schäffer L (2008) Activation of the insulin receptor (IR) by insulin and a synthetic peptide has different effects on gene expression in IR-transfected L6 myoblasts. J Biol Chem 412: 435-445.

4. Kurtzhals P, Schaffer L, Sorensen A, Kristensen C, Jonassen I, et al. (2000) Correlations of receptor binding and metabolic and mitogenic potencies of insulin analogs designed for clinical use. Diabetes 49: 999-1005.

5. Schäffer L (1994) A model for insulin binding to the insulin receptor. Eur J Biochem 221: 1127-1132.

6. Schaffer L (2006) Pharmaceutically active insulin receptor-modulating molecules. PCT Int Appl WO2006018450.

7. Schäffer L, Brissette RE, Spetzler JC, Pillutla RC, Ostergaard S, et al. (2003) Assembly of high-affinity insulin receptor agonists and antagonists from peptide building blocks. Proc Natl Acad Sci USA 100: 4435-4439. 
Citation: Mesonzhnik N, Krotov G and Appolonova G (2017) Mass Spectrometry Characterization of a Novel Insulin Mimetic Peptide s597. Pharm Anal Acta 8: 549. doi: 10.4172/2153-2435.1000549

Page 6 of 6

8. Varewijck AJ, Janssen JA (2012) Insulin and its analogues and their affinities for the IGF1 receptor. Endocr Relat Cancer 19: F63-F75.

9. Vigneri R, Squatrito S, Frittitta L (2014) Selective insulin receptor modulators (SIRM): a new class of antidiabetes drugs? Diabetes 61: 984-985.
10. https://www.wada-ama.org/sites/default/files/resources/files/ 2015_wada_anti-doping_testing_figures_report_0.pdf

11. https://www.wada-ama.org/sites/default/files/resources/files/ 2016-09-29_-_wada_prohibited_list_2017_eng_final.pdf 